\title{
Ueber das Gleichgewicht Gelatine-Salzsäure. II.
}

\author{
Von Robert Wintgen und Heinz Vogel (Göttingen).
}

(Eingegangen ain 9. Oktobar 1921.)

In einer Untersuchung über das Gleichgewicht Gelatine-Salzsäure haben R: Wintgen und K. K r ïg e ${ }^{1}$ ) festgestellt, dab sich aus dem Salzsăurebindungsvermögen der Gelatine für diese ein Verbindungsgewicht von 839 ergibt. Die Untersuchungen waren bei einer Salzsăurekonzentration von 0,05 und 0,1 ausgeführt worden, also in Konzentrationsgebieten, wo es möglich war, daß bereits eine ganze Anzahl der in der Gelatine vorhandenen Amidogruppen mit der Salzsăure reagierten, wonach aiso das Molekulargewicht der Gelatine ein Vielfaches von 839 sein mübte. Auf ein weit höheres Molekulargewicht als 839 lieBen vor allem auch die Art und $Z$ ahl der aus der Gelatine von L. L. van Slyke ${ }^{2}$ ) und H. D. Dakin ${ }^{3}$ ) gewonnenen Abbauprodukte und das typische kolloide Verhalten der Gelatine schlieben. Anderseits sprachen eine Reihe nicht ohne weiteres $2 \mathfrak{u}$ vernachlässigende Anzeichen dafür, dab der Gelatine in schwachsaurer oder heiber Lösung ein Molekulargewicht von ungefähr 900 zuzusprechen sei. (R. Wintgen und K. Krüger, loc. cit.) Weiteres Material zur Klärung dieser Frage beizubringen, wat die Hauptaufgabe dieser Untersuchung. Nach den wohl allgemeinen Vorstellungen über die Stufendissoziation besonders bei schwachen mehrsäurigen Basen treten erst bei relativ hoher Säurekonzentration mehrere basische Gruppen in Reaktion, bei ganz geringer Säurekonzentration vollzieht sich die Salzbildung aber nur an e ine $r$ basischen Gruppe. Wenn nun die Gelatine in der oben erwähnten Salzsäurekonzentration $(0,05-0,1)$ bereits mit mehreren Amidogruppen reagiert hat, so ist dies demnach ziemlich ausgeschlossen, wenn es sich um Lösungen mit ganz niedriger Wasserstoffionenkonzentration handelt. Es muB sich also bei der Untersuchung ganz schwachsaurer Gelatine-Salzsäurelósungen ein anderes Verbindungsgewicht ergeben, das Säureverbindungsvermögen müfte also in diesen Gebieten durch eine ganz andere Gleichung als durch die in

1) R. Wintgen u. K. Krüger, Koll.-Zeitscirr. 28, 81 (1921).

2) L. L. van Slyke, Journ. of biol. chem. 10, 15 (1911), s. auch D. J. Lloy d, Biochem. Journ. 14, 147 (1920). (1920). der obigen Arbeit angewandte, seinen Aus. druck finden. Deshalb lag es im Plan unserer Arbeit, die Wasserstoffionenkonzentration bis zu sehr niedrigen Werten zu verfolgen und $z u$ prüfen, ob sich hier ein höheres Verbindungsgewicht ergeben würde. Weiter sollte untersucht werden, ob die so gewonnenen Vorstellungen über die Zusammensetzung von Gelatine-Salzsäuregemischen durch Leitfähigkeitsmessungen bestätigt würden. Ferner schien es uns nicht ausgeschlossen, durch Membranfiltration Anhaltspunkte für eine Beurteilung der chemischen Einheitlichkeit der Gelatine zu gewinnen. Endlich sollte auch das aus Gelatine durch langes Kochen mit Wasser entstehende nicht mehr gelatinierende $\beta$-Glutin vergleichsweise untersucht werden.

Die in der 1. Mitteilung den Berechnungen zu Grunde gelegte Gleichung für das Gleich. gewicht Gelatine-Salzsäure:

$$
\begin{array}{r}
{\left[H^{+}\right]=-\frac{1}{2}\left(\frac{m}{M}-n+K\right)} \\
+\sqrt{\frac{1}{4}\left(\frac{m}{M}-n+K\right)^{2}+K n}
\end{array}
$$

hatte für $M$ den Wert 1014 und für $K$ den Wert $4,139 \cdot 10^{-4}$ ergeben. Hierbei war $K$ nur aus einem kleinen Kurvenstück, also nur sehr ungenau bestimmt worden; im Gebiete stärkerer Hydrolyse, also bei geringer Wasserstoffionenkonzentration, könnte ein genauerer Wert von $\mathrm{K}$ ermittelt werden, ein weiterer Grund, die Messungen auch in ganz schwach salzsauren Lösungen vorzunehmen. $\mathrm{Da}$ nach der früher angewandten Methode der Methylazetat-Verseifung die Messungen bei geringer Wasserstoffionenkonzentration sehr zeitraubend und ungenau sind, bedienten wir uns jetzt der potentiometrischen Methode und prüften hiermit gleichzeitig einige der früheren Messungen.

$$
\text { Versuchsanordnung. }
$$

Die Herstellung der Lösungen geschah wie in Mitteilung $\mathrm{I}$. Alle Versuche wurden bei $25,0^{\circ} \mathrm{im}$ Thermostaten ausgeführt.

Der Wasserstôff war der Bombe entnommen und sorgfältig gereinigt. Die Messung geschah in der üblichen Weise unter fortwährendem Durchleiten von Wasserstoff, nachdem die Elektroden vor dem Einführen der Flüssigkeit mit Wasserstoff gesăttigt waren. Die Lösungen 
mit 30 und $40 \mathrm{~g}$ Gelatine waren während der Messung sehr zähflüssig, die mit $70 \mathrm{~g}$ zu einer Gallerte erstarrt, deshalb war das Sättigen der Elektroden mit Wasserstoff mit Schwierigkeiten verbunden. Die Anordnung der Schaltung war die übliche.

Als Halbelektrode diente eine sorgfăltigst mit den reinsten Materialien hergestellte $1 /$ ro n K Cl-Kalomelelektrode. Die Wasserstoffionenkonzentration wurde berechnet nach der Formel

$$
\left[H^{+}\right]=10-\frac{\pi-0,3369}{0,05912}
$$

hierin bedeuten $\pi$ das Potential der gesamten, 0,3369 das Potential einer ähnlichen Kette, wenn die Elektrodenflüssigkeit an der PlatinWasserstoffelektrode die Wasserstoffionenkonzentration 1 hat [s. P. L. Sörensen ${ }^{4}$ ), Loomis und $A c r e e^{5}$ ) und $A$ uerbach $\left.\left.{ }^{6}\right)\right]$.

$$
0,05912=\frac{R \cdot F}{n \cdot F \cdot 0,4343}
$$

Das Westonelement wurde mit einem Nor: malelement von der Reichsanstalt verglichen und hatte die genaue E. M.K. 1,0186 bei $20^{\circ}$. Als Nullinstrument diente ein Kapillarelektrometer; alle im Thermostaten stehenden GefäBe waren zwecks Isolation mit einer dünnen Paraffinschicht überzogen; bei den hochkonzentrierten Gelatinelösungen, welche einen groben Widerstand besitzen, wurde bei der Feineinstellung mit offenem Hahn gemessen. Die Gefahr der Vermischung war hier wegen $\mathrm{Er}^{-}$ starrung der Gelatinelösung äuBerst gering. Wie wir uns des öfteren überzeugten, wurde durch längeres Oeffnen des Hahnes das Potential nicht geändert. Trotzdem lieb auch so die Genauigkeit der Einstellung noch zu wünschen übrig. Schon P. L. Sörensen (loc. cit.) weist darauf hin, dah die elektromotorische Kraft von proteinhaltigen Gasketten nicht so scharf wie sonst $z \mathfrak{u}$ ermitteln ist.

Bei der Berechnung der Wasserstoffionenkonzentration bietet vor allem die Ausschaltung der Zwischenschicht gewisse Schwierigkeiten. Nach N. Bjerrum ${ }^{7}$ ) läBt sich das Diffusionspotential durch Extrapolation der mit $3,5 \mathrm{n}$ Kaliumchlorid- und 1,75 n Kaliumchloridlösung

4) S.P. L. Sơ rense n, Ergebrisse d. Physiol. 12, 415 (1912).

5) N. E. Loom is u. S. F. Acree, Amer. Chem. Journ. 46, 585, 621 (1911).

6) F. A ue rbach, Zeitschr. f. Elektr. 18, 13 (1912).

7) N. B.jerrum, Zeitschr. f. physik. Chem. 53, 428 (1905); Zeitschr. f. Elektr. 17, 389, 581 (1911). als Zwischenschicht gewonmenen Werte eliminieren. Loo $\mathrm{m}$ is und $\mathrm{Acree}$ (loc. cit.) fanden dagegen, daß schon durch gesättigte, also $3,5 \mathrm{n}$ Kaliumchloridlösung das Diffusionspotential fast vollständig vernichtet wird. Wir haben, wie jetzt meist üblich, den extrapolierten Wert benuitzt.

\section{Versuchsergebnisse.}

Die Messungen sind in folgender Tabelle I zusammengestellt.

Tabelle I.

Wasserstoffionenkonzentration von GelatineSalzsäure - Gemischen bei $25,0^{\circ}$.

\begin{tabular}{l|c|c|l|l|l}
\hline $\mathbf{n}$ & $\mathrm{m}$ & $\boldsymbol{\pi}$ & {$[\mathrm{H}+]_{\text {gef. }}$} & {$[\mathrm{H}+]_{\text {ber. }}$} & {$[\mathrm{H}+]^{\prime}$ ber. } \\
\hline \hline 0,05 & 0 & 0,4140 & 0,0496 & 0,0500 & 0,0500 \\
0,05 & 1 & 0,4139 & 0,0498 & 0,0490 & 0,0491 \\
0,05 & 30 & 0,4332 & 0,0235 & 0,0210 & 0,0222 \\
0,05 & 40 & 0,4508 & 0,0118 & 0,0119 & 0,0131 \\
0,05 & 70 & 0,5270 & 0,000602 & 0,00102 & 0,000590 \\
0,02 & 0 & 0,4375 & 0,0199 & 0,0200 & $\mathbf{0 , 0 2 0 0}$ \\
0,02 & 2,5 & 0,4398 & 0,0182 & 0,0176 & 0,0177 \\
0,02 & 10 & 0,4520 & 0,0113 & 0,0105 & 0,0108 \\
0,004 & 10 & 0,5661 & 0,000135 & 0,000261 & 0,000135
\end{tabular}

Darin bedeutet $\mathrm{n}$ die Normalität der Salzsäure im Gemisch, in die $\mathrm{g}$ Gelatine (lufttrocken) im Liter, $\pi$ die elektromotorische Kraft der gesamten Kette, $\left[\mathrm{H}^{+}\right]$die daraus nach Formel (2) berechnete Wasserstoffionenkonzentration. Unter $\left[\mathrm{H}^{+}\right]_{\mathrm{ber}}$, finden sich die nach Formel (1) mit den in der 1. Mitteilung angegebenen Werten $M=1014$ und $K=4,139 \cdot 10^{-4}$ berechneten Werte. Diese Werte wurden, wie eingangs erörtert, seinerzeit gewonnen durch die Ermittlung der Methylazetatverseifung in Gelatine-Salzsäurelösung in einem Gebiet, wo nur geringe Hydrolyse auftrat, demgemäß war der so ermittelte Wert von $\mathrm{K}$, worauf schon in der I. Mitteilung hingewiesen wurde, ziemlich ungenau. Daher kommt es auch, daß die mit dieser Konstanten berechneten Wasserstoffionenwerte in obiger Tabelle gegen die potentiometrisch experimentell ermittelten vielfach erheblich abweichen.

Es wurde deshalb versucht, aus den vorliegenden Messungen, die, wie schon erwähnt, absichtlich im Gebiet starker Hydrolyse ausgeführt wurden, einen richtigeren Wert für $K$ zu errechnen. Hierzu wurden, wie in der I. Mitteilung, zusammengehörige Werte von $\left[\mathrm{H}^{+}\right]$und $\frac{\mathrm{m} \cdot[\mathrm{H}+]}{\mathrm{n}-\left[\mathrm{H}^{+}\right]}$in ein Koordinatensystem eingezeichnet und zwar vor allem diejenigen 
Messungen benutzt, die im Gebiete starker Hydrolyse liegen. (Siehe Fig. 1.)

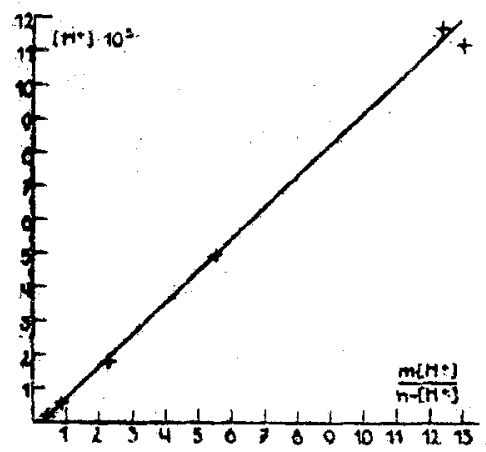

Fig. 1

$[\mathrm{H}+]$ in Abhängigkeit von $\frac{\mathrm{m} \cdot[\mathrm{H}+]}{\mathrm{n}-[\mathrm{H}+]}$ in GelatineSalzsäurelosungen bei $25,0^{\circ}$.

Die Wertepaare liegen wieder auf einer Geraden (der letzte Wert aus $\mathrm{m}=10 ; \mathrm{n}=0,02$; $\left[\mathrm{H}^{+}\right]=0,0113$ ergibt rechnerisch nur einen relativ. ungenauen Wert für $\mathrm{n}-[\mathrm{H}+]$; er liegt wohl aus diesem Grunde etwas abseits von der Geraden). Berechnet aus den Werten

\begin{tabular}{c|c|c}
\hline $\mathrm{m}$ & \multicolumn{1}{|c|}{$\mathbf{n}$} & \multicolumn{1}{|c}{$[\mathrm{H+}]$} \\
\hline 50 & 0,05 & 0,00498 \\
10 & 0,004 & 0,000135
\end{tabular}

ergibt sich die Gleichung

$[\mathrm{H}+]=\frac{1}{1069,5} \cdot \frac{\mathrm{m} \cdot[\mathrm{H}+]}{\mathrm{n}-\left[\mathrm{H}^{+}\right]} 0,0001916$; daraus

(3) $[\mathrm{H}+]=-\frac{1}{2}\left(\frac{\mathrm{m}}{1069,5}-\mathrm{n}+0,0001916\right)$

$+\sqrt{\frac{1}{4}\left(\frac{m}{1069,5} r+0,0001916\right)^{2}+n .0,0001916}$.

Nach dieser Formel (3) sind die in Tabelle I unter $\left[\mathrm{H}^{+}\right]^{\prime}$ ber. angegebenen Werte berechnet; sie stimmen gut mit den gefundenen überein. Ebensogut ist die Uebereinstimmung mit den Werten in der I. Mitteilung. Entsprechend der Annahme, daB die in diesen Gemischen enthaltene freie Salzsäure in allen Konzentrationen völlig zerfallen ist, wurden die $\mathrm{R}$-Werte für die Verseifungskonstante aus der $\mathrm{l}$. Mitteilung in den Mischungen mit $0,1 \mathrm{n}$ Salzsäure zur Ermittlung von $[\mathrm{H}+]$ mit demselben Faktor $(345,8)$ multipliziert, wie in den Mischungen mit 0,05 n Salzsäure. Die Uebereinstimmung zwischen den gefundenen und berechneten Werten ist jetzt überraschend gut, auch die Messungen mit 0,1 п Salzsäure, die beträchtlich nach einer Seite abwichen, werden durch die Formel (3) sehr gut wiedergegeben. Die Uebereinstimthung ist um so befriedigender, wenn man die Schwierigkeiten bei den Messungen berücksichtigt. Die Schwierigkeiten bestehen einmal darin, bei hochkonzentrierten Gelatinelösungen mit Wasserstoff völlig zu sättigen, anderseits an der bereits erwähnten Unsicherheit in der Elimination des Diffusionspotentials ${ }^{8}$ ). Eine $\mathrm{Zu}$ sammenstellung aller Messungen gibt Tabelle II.

Ein Anzeichen dafür, daß bei niederen Säurebzw. Wasserstoffionenkonzentrationen. weniger basische Valenzen der Gelatine in Reaktion treten als bei hoher Säurekonzentration, ist aus den Zahlen nicht zu bemerken. $\quad E s$ ist im Gegenteil hiernach wohl als erwiesen anzusehen, dafi, wenn wir die Gelatine zunächst einmal als chemisch einheitliche Substanz annehmen, nur eine Amidogruppe in dem untersuchten Konzentrationsbereich mit der Salzsäure in Reaktion tritt. Die Gelatine verhält sich also bei Gegenwart von verdünnter Salzsäure wie eine normale einsäurige Base mit dem Mòlekulargewicht ${ }^{9}$ ) 1070 bezogen auf lufttrockene oder 885 bezogen auf wasserfreie Gelatine - und der basischen Dissoziationskonstante

$$
\mathrm{K}_{\mathrm{b}}=\frac{1,1 \cdot 10^{-4}}{1,916 \cdot 10^{-4}}=5,74 \cdot 10^{-11} \text {. }
$$

Die in wässeriger Lösung bei gewöhnlicher Temperatur typisch kolloide Gelatine erfährt also bei Gegenwart von verdünnter Salzsäure (und wahrscheinlich auch bei höheren Temperaturen) eine Desaggregation, eine Art Peptisation, wahrscheiniich bis zu den Molekülen im chemischen Sinne. Hierfür spricht auch das Verhalten von Gelatinelösungen beim isoelektrischen Punkt, von dem noch die Rede seín wird.

8) Bei der potentiometrischen Erimitteling der Wasserstoffionenkonzentration kann, wie erwähnt, nach Sörepsen mit keiner größeren Genauigkeit als \pm 3 Millivolt gerechnet werden; die Abweichungen in Tabeile $11 z$ wischen den gefundenen und berechneten Werten sind bedeutend geringer.

9) Vgl. hierzu die Untersuchungen. von P. Ka r rer und seinen Mitarbeitern über Polysaccharide [Helv. chim. Acta 3, 620 (1920); 4, 185, 249, 263 (1921); Naturw. 9, 399], der Methylierungsprodukte der Stärke und des Innulins darstelite. Er fand, daB diesen Produkten in Lösungen von Wasser, Phenol und Bromo: form Molekulatgewichte von 1000 bis 2000 zukommen; da durch das Methylieren kein Abbau stattfindet, müssen die Molekulargewichte der Stärke und des Innulins nur etwas kleiner sein. Ka r re $\mathrm{t}$ fand ferner [Helv. chim. Acta 4, 811 (1921)], daB Diamylose, $\alpha-$ Tetramylose, $\beta$-Hexamylose, $\alpha$-Oktamylose und „165liche Stärke nach Zulkowsky" mit Natronlauge wasserlösliche Additionsverbindungen derselben $\mathrm{Zu}$ sammensetzung $\left(\mathrm{C}_{12} \mathrm{H}_{20}, \mathrm{O}_{20}, \mathrm{NaO} \mathrm{H}\right) \mathrm{n}$ liefern. 
Tabelle II.

Wassersioffionenkonzentration in Gelatine-Salzsäuregemischen bei $25,0^{\circ}$. n und $\mathrm{m}$ : die Mole Salzsäure und $g$ Gelatine im Liter. $\left[\mathrm{H}^{+}\right]$berechnet nach Formel (3).

\begin{tabular}{|c|c|c|c|c|}
\hline \multirow{3}{*}{$\mathrm{n}$} & \multirow{3}{*}{ m } & \multicolumn{3}{|c|}{$[\mathrm{H}+]$} \\
\hline & & \multicolumn{2}{|c|}{ gemessen } & berechnet \\
\hline & & kstalytiseh & potentiometrisch & \\
\hline 0,1 & 0 & 0,0985 & & 0,1000 \\
\hline 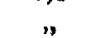 & 5 & 0,0952 & & 0,0953 \\
\hline " & 10 & 0,0909 & & 0,0907 \\
\hline$"$ & 20 & 0,0829 & & 0,0813 \\
\hline$"$ & 25 & 0,0768 & & 0,0767 \\
\hline " & 30 & 0,0710 & & 0,0720 \\
\hline$"$ & 40 & 0,0635 & & 0,0627 \\
\hline " & 50 & 0,0549 & & 0,0534 \\
\hline 0,05 & 0 & 0,0500 & 0,0496 & 0,0500 \\
\hline ", & 1 & 0,0492 & 0.0498 & 0,0491 \\
\hline " & 2 & 0,0482 & & 0,0481 \\
\hline$"$ & 5 & 0,0452 & & 0,0453 \\
\hline " & 10 & 0,0410 & & 0,0407 \\
\hline ", & 20 & 0,0306 & & 0,0314 \\
\hline ", & 30 & 0,0207 & 0,0235 & 0,0212 \\
\hline 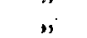 & 40 & 0,0117 & 0,0118 & 0,0131 \\
\hline & 50 & 0,00498 & & 0,00498 \\
\hline$"$ & 60 & 0,00180 & & 0,00127 \\
\hline & 70 & & 0,000602 & 0,000590 \\
\hline 0,02 & 0 & & 0,00199 & 0,0200 \\
\hline$"$ & 2,5 & & 0,0182 & 0,0177 \\
\hline 0,0004 & $\begin{array}{l}10 \\
10\end{array}$ & & $\begin{array}{l}0,0113 \\
0,000135\end{array}$ & $\begin{array}{l}0,0108 \\
0,000135\end{array}$ \\
\hline
\end{tabular}

Die Berechnung wurde auch nach der Formel von P. L. Sörensen (R. Wintgen und $\mathrm{K}$. Krüger, loc. cit. 84) durchgeführt, also unter der alten Annahme des nur teilweisen Zerfalls der freien Salzsäure und des gebildeten "Gelatinechlorids" Auf diese Weise konnte aber eine so gute Uebereinstimmung $z$ wischen den gefundenen und berechneten Werten nicht erzielt werden $\left.{ }^{10}\right)$.

Bestimmung des isoelektrischen Punktes von Gelatinelösungen.

Für die Anwendbarkeit der entwickelten Vorstellungen sprechen auch folgende Beobachtungen.

Bei Schüttelversuchen, von denen weiter unten noch die Rede sein soll, hatte es sich gezeigt, daß eine nicht geschüttelte halbpro-

10) Von einer Mitteilung des umfangreichen Zahlenmaterials haben wir geglaubt absehen zu sollen; dieses findet sich in der ausfuthrlichen Dissertation: H. Vogel, Ueber das Salzsăurebindungsvermogen der Gelatine und des $\boldsymbol{\beta}$-Glutins, ein Beitrag zur physikalischen Chemie und Kolloldchemie der Gelatine (Gottingen 1921). zentige und in bezug auf Salzsäure $1 / 1000 \mathrm{n}$ Gelatinelösung nach mehrstündigem Stehen eine auffallende Trühung aufwies. Um diese Erscheinung năher zu studieren, versetzten wir in einer Reihe von Reagenzgläsern Gelatinelösungen wechselnder Konzentration mit wechselnden Mengen Salzsäure und zwar in einem Vorversuche je $9 \mathrm{ccm}$ einer $0,222-\mathrm{bzw}$. 0,555 prozentigen Gelatinelösung mit je $1 \mathrm{ccm}$ Salzsäure verschiedener Normalität: $1,0,0,5$, $0,2,0,1,0,02,0,01,0,005,0,001 \mathrm{n}$, so daß die Lösung in bezug auf Salzsäure 0,1 , $0,05,0,02,0,01,0,002,0,001,0,0005$ und $0,0001 \mathrm{n}$ und in bezug auf Gelatine $0,2-\mathrm{bzw}$. 0,5 prozentig waren. Nach mèrstindigem Stehen traten Trübungen aut, die sich mit der Zeit zu nicht unbeträchtlichen Mengen Sediment vermehrten und zwar in der 0,2 prozentigen Gelatinelösung bei einer Salzsäurekonzentration vor 0,0005 , in der 0,5 prozentigen Losung in dem Gebiet von 0,002 bis 0,0005 ; bei 0,001 lag im letzteren Falle das Tribungsmaximum.

Beim Erwärmen auf ungefähr $30-40^{\circ}$ verschwanden die Trübungen, beim Erkalten nach längerem Stehen traten sie wieder auf,

Nachdem wir so roh das Maximum der Trübung bestimmt hatten, legten wir nun zur genaueren Bestimmung seiner Lage die Salzsäurekonzentration innerhalb engerer Grenzen fest. Zu diesem $Z$ weck stellten wir SalzsäureGelatinegemische her, in denen die Konzentration der Säure $1 / 1800,1 / 1900,1 / 2000$ usw. bis $1 / 3300$ betrug. Nach kurzer Zeit $(15 \mathrm{Mi}$ nuten) traten bereits die Trübungen auf und nach ungefähr 24 Stunden war das Maximum bei deutlich $1 / 2700$ n Salzsäure zu erkennen. Die Lage des Maximums ist abhängig von der Temperatur. Deshalb wurden die Versuche nun nochmals im Thermostaten bei genau $25,0^{\circ}$ ausgeführt. Für die 0,2 prozentige Gelatinelösung lag der Punkt stärkster Trübung bei $1 / 3000 \mathrm{n}$ Salzsäure, für die 0,5 prozentige bei $1 / 1300$ n Salzsäure.

Es lag die Vermutung nahe, dab das Flokkungsmaximum im isoelektrischen Punkte der Gelatine aufgetreten sei ${ }^{11}$ ). Wir berechneten daher mit Formel 9 aus der Menge der Gelatine und Salzsäure die Wasserstoffionenkonzentration des Gemisches mit der stärksten Trübung. Unter der Voraussetzung der vollständigen Dissoziation

11) Vgl. hierzu die Untersuchungen von J. Loeb (Journ. of the Gen. Physiol. 1, 365, 1919), die uns als verspätet eingelaufene Auslandsliteratur erst nach $\mathbf{A b}$ schluB unserer Untersuchungen zuganglich wurden. 
der Salzsăure und des "Gelatinechlorids" ergaben sich folgende Werte:

\begin{tabular}{c|c|c|c}
\hline t Grad & $\begin{array}{c}\text { Geiatine } \\
\text { Proz. }\end{array}$ & $\begin{array}{c}\text { Konz. der HCl } \\
\text { im Gemisch }\end{array}$ & {$\left[\mathrm{H}^{+}\right]$} \\
\hline 25,0 & 0,2 & $1 / 3000$ & $3,58.10-5$ \\
25,0 & 0,5 & $1 / 1300$ & $3,57.10^{-5}$
\end{tabular}

Bei $18^{\circ}$ wurde im Ueberführungsapparat von L. Michaelis und W. Grineff 12 ) der isoelektrische Punkt des Glutins bei einer Wasserstoffionenkonzentration von $1,6-3,510^{-5}$ gefunden und später yon Wo. $\mathrm{Pauli}$ und M. S a m e c ${ }^{\text {t3 }}$ ) bestätigt.

Unter Berücksichtigung der verschiedenen Temperaturen ergibt sich also eine sehr gute Uebereinstimmung ${ }^{14}$ ).

Aus der Anwendbarkeit unserer Formel bis zum isoelektrischen Punkt geht wiederum hervor, dab die Gelatine in dem untersuchten Konzentrationsintervall bis hinauf $z u$ o, $1 \mathrm{n}$ Salzsäure nur mit einer Amidogruppe reagiert, es sei denn, dak das, was wir über Stufendissoziation bei anderen Stoffen wissen, bei Gelatine $n$ icht zutrifft.

Wenn die entwickelten Vorstellungen richtig sind, so mübten auch Gelatinelösungen hoher Konzentration bei passendem Salzsäurezusatz ausflocken; eine dreiprozentige Gelatinelösung nach der oben entwickelten Formel bei einer Konzentration der Salzsäure von 0,001384. Bei einem in diesen Mengenverhältnissen angestellten Versuch entstand in der Tat in der anfangs völlig klaren Gallerte nach etwa einer halben Stunde eine Trübung; nach längerem Stehen war durch die ganze Gallerte eine Anzahl feiner diskreter Pünktchen aufgetreten; die Erscheinung erinnerte an das Bild, welches man bei der Kristallisation mit Kristallisationszentren zu sehen gewohnt ist.

Die im isoelektrischen Punkte befindlichen Lösungen von 0,2 und 0,5 Proz. Gelatine mit $1 / 3000$ und $1 / 1300$ n Salzsäure wurden weiter im Zs ig mondy'schen Immersionsultramikroskop bei Sonnenlicht untersucht. Die Trübungen bestanden aus zahilreichen, lebhaft bewegten, aufleuchtenden Einzelteilchen, außerdem waren flockenartige Gebilde aus vielen Einzelteilchen vorhanden, die als Ganzes eine minimale Bewegung aufwiesen, in diesen waren die Einzel-

12) L. Michaelis u. W. Grineff, Biochem. Zeitschr. 41, 373 (1912).

13) Wo. Pauli Kolloidchemie der Eiweißkörper, 1. Halfte (Dresden 1920), 30 .

14) Aus $K_{W}^{18^{\circ}}=0,58$ und $K_{W}^{25^{\circ}}=1,1 \cdot 10^{-14}$, witden sich aus unseren Messungen 2,5 $10^{-6}$ tür $18^{\circ}$ ergeben. teilchen noch deutlich zu erkennen, and bei genauem Zusehen und bester Beleuchtung kann man feststellen, daB die Einzelteilchen innerhalb der Flocken noch eine geringe Eigenbewegung besitzen. Von diesen Flocken lösten sich manchmal kleine Petzen $a b$ und lagerten sich an andere Flocken wieder an. Nach fünftägigem Stehen waren sämtliche Einzelteilchen verschwunden und nur noch Flocken vorhanden. Frisch hergestellte, also noch nicht gelatinierte Lösungen mit $0,5-1$ Proz. Gelatine ohne Salzsäurezusatz zeigen im Ultramikroskop höchstens einen ganz schwachen diffusen Lichtkegel. Läbt man aber halbprozentige Gelatinelösungen ohne Salzsäurezusatz längere Zeit stehen, so kann man in Ultramikroskop ganz ähnliche Erscheinungen wie die oben beschriebenen beobachten ${ }^{15}$ ). Makroskopisch sichtbare grobe Trübungen bzw. Niederschläge treten aber erst bei Zusatz von Salzsäure auf.

\section{Die Leitfähigkeit von Gelatine-Salz- säurelösungen.}

Die Mekmethode war die übliche. Für die Gemische mit $0,05 \mathrm{n}$ und $0,025 \mathrm{n}$ Salzsăure ergeben sich die Werte der Tabelle III bzw. Fig. 2.

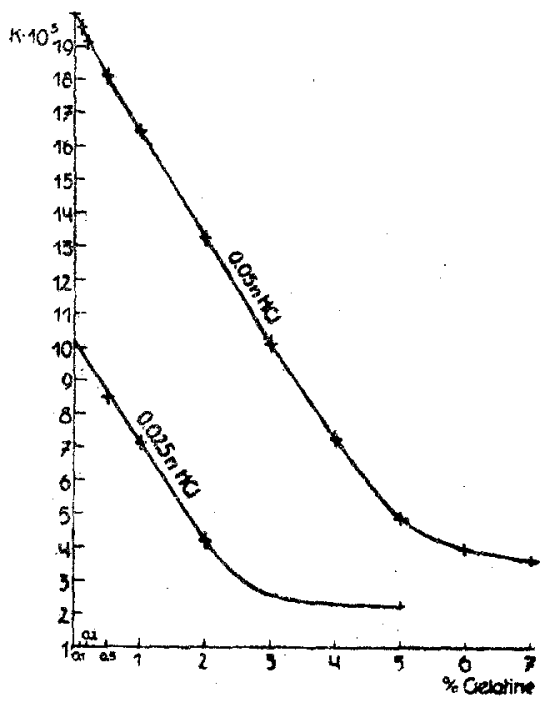

Fig. 2

Spezifische Leitfăhigkeit von Gelatine- $0,05 \mathrm{n}$ und $0,025 \mathrm{n} \mathrm{Hi} \mathrm{Cl}$ - Gemischen in Abhangigkeit vom ProzentGehalt der Gelatine bel $25,0^{\circ}$.

15) R. Zsigmondy, Kolloidchemie (3. Aufl. 1920), 365 ; W. B a chman n, Zeitschr. t. anorg. Chem. 73, 125 (1911); W. Menz, Zeitschr. \&. physik. Chem. 66. 129 (1909); 'P. v. Weimarn, Koll.-Zeitschr. 4, 133 (1909); 6, 277 (1910). 
Tabelle III.

Spezifische Leithathigkeit $*$ von Gelatine- $0,05 \mathrm{n}$ und $0.025 \mathrm{n} \mathrm{HCl}$-Gemischen in Abhängigkeit vom Gelatinegehalt. ( $m=g$ Gelatine in Liter) bei $25,0^{\circ}$.

\begin{tabular}{c|c|r|r|r}
\hline \multicolumn{2}{c||}{$0,05 \mathrm{n} \mathrm{HCl}$} & \multicolumn{3}{|c}{$0,025 \mathrm{n} \mathrm{HCl}$} \\
\hline $\mathbf{m}$ & $x \cdot 10^{3}$ & $\mathrm{~m}$ & $\times .103$ & $\left.\mathrm{y}^{\mathbf{1 6}}\right)$ \\
\hline 0 & 19,94 & 0 & 10,06 & 0,02500 \\
1 & 19,53 & 5 & 8,59 & 0,02037 \\
2 & 19,12 & 10 & 7,11 & 0,01576 \\
5 & 18,10 & 20 & 4,17 & 0,006812 \\
10 & 16,52 & 50 & 2,27 & 0,000216 \\
20 & 13,25 & & & \\
30 & 10,12 & & & \\
40 & 7,28 & & & \\
50 & 4,84 & & & \\
60 & 3,983 & & & \\
70 & 3,660 & & &
\end{tabular}

Bezeichnet man mit y die Konzentration der ungebundenen, mit $n$ wie vorher die Konzentration der gesamten Säure, mit $\alpha$ und $\rho^{\prime \prime}$ die Dissoziationsgrade der Salzsäure und des "Gelatinechlorids", so ist unter der Voraus. setzung, $\mathrm{daB}$ sich die Leitfähigkeit $x$ additiv zusammiensetzt

$$
1000 x=\mathrm{y} \cdot \alpha \cdot A_{\infty}^{\mathrm{HC}}+(\mathrm{n}-\mathrm{y}) \beta^{\prime} A_{\infty}^{\mathrm{GeCl}}
$$

(4) $1000 x=\mathrm{y}\left(\alpha_{\infty}^{\mathrm{HCl}}-\beta^{\prime} A_{\infty}^{\mathrm{GeCl}}\right)+\beta^{\prime} \mathrm{n} A_{\infty}^{\mathrm{GeOl}}$.

$\mathrm{Da}$ in einer Lösung, welche zwei Elektrolyte mit einem gemeinsamen Ion enthält, der Dissoziationsgrad jedes Elektrolyten durch die Gesamtkonzentration des gemeinsamen lons bestimmt ist, und diese bei konstantem $n$ sich nicht ändert, so sind in unserem Falle $\alpha$ und $\beta^{\prime}$ bei den Versuchen mit $0,05 \mathrm{n} \mathrm{HCl}$ und $0,025 n \mathrm{HCl}$ jeweils konstant. Danach müBte $1000 *$ linear von y abhängen. Dieses wird durch Fig 3 bestätigt, wo für y die Werte nach Formel (3) eingesetzt sind ${ }^{17}$ ).

16) Berechnet nach Formel (3). Die y-Werte futr $0,05 \mathrm{n} \mathrm{HCl}$ finden sich in Tabelle II.

i7j Bekanntlich stimmt die Wasserstoffionenkonzentration, wie man sie aus Leitfăhigkeitsmessungen errmittelt, nicht überein mit der nach katalytischen und potentiometrischen Methoden gewonnenen. [N. Bjerru m, Zeitschr. f. anorg. Chem. 109, 275. (1920); Ghosh, Zeitschr. f. phyik. Chem. 98, 211 (1921)]. Wir haben geglaubt, für unsere Zwecke die katalytisch und potentiometrisch ermittelte Wasserstoffionenkonzentration für die Berechnung der Leitfähigkeit gleich der Konzentiation der freien Saure setzen zu düfen, da nach den Messungen det Tabelie II sowohl die $0,05 \mathrm{n}$ wie die $0,02 \mathrm{n} \mathrm{HCl}$ vollstezndig dissoziiert scheint.

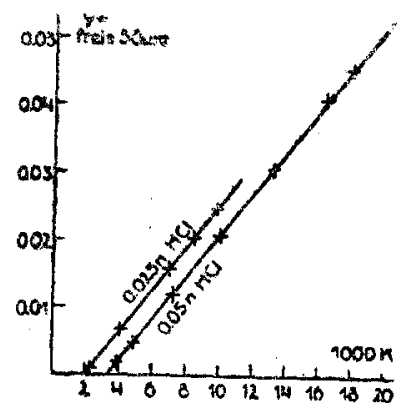

Fig. 3

Spezifische Lcitfähigkeit von Gelatine- $0,05 n$ and $0,025 \mathrm{n}$ Salzsăuregemischen in Abhängigkeit von der Konzentration der treien Säure bei 25,00.

Dis Gleichung der beiden Geraden, abgeleitet aus den in der folgenden Zusammenstellung angegebenen Werteparen, ergibt im Mittel $A_{\infty}^{\mathrm{EC}}=425,5$ in Uebereinstimmung mit den sonst hierfür angegebenen Werten, für $A_{\infty}^{\text {Genl }}=88,5$, woraus sich 13 für die Wanderungsgeschwindigkeit des (Gelatineions ${ }^{18}$ ) ergeben würde. Die Werte für $\alpha$ ergeber sich sus unseren Messungen und denen von Bray und $\mathrm{Hunt}{ }^{19}$ ), die Werte für $\beta^{\prime}$ sind mit P. L. Sörensen ${ }^{20}$ gleich denen des Ammoniumchlorids in derselben Verdünnung gesetzt. (Näheres siehe Vogel, loc. cit:) Durch die Leitfähigkeitsmessungen werden also die aus der Messung der Wasserstoffionenkonzentration gewonnenen Resultate bestätigt.

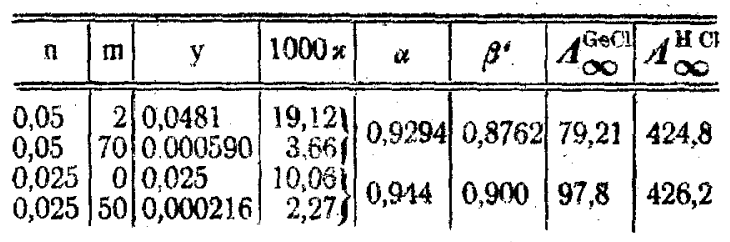

Die Berechnung besonders der Wanderungsgeschwindigkeit des Gelatineions kann schon

18, Nach G. Bredig [Zeitschr f. physik. Chem. 13, 191 (1894)] nähert sich die Wanderungsgeschwindigkeit der Ionen mit zunehmender Atomzah dem Gienzwert 17-20. Wo. Pauli [Kolloidchemie der Eiweifkörper, 1. Hälfte (Dresden 1920), 108] tindet $C_{\text {Albumin }}$ $=5-8$ aus dem Diffusionspotential, W. B. Hardy [Journ. of Physiol. 33, 251 (1905)] aus der Verschie. bung der Grenzschicht unter dem Einfluß eines Poten tialgefalles nach der Methode von W. C. D, Whetham 9-11,5 bzw. 7,7 für Globulinionen. T. B. Roberts on [Journ. of physiol. Chern. 11, 542 (1907) 12, 473 (1908] land rund 7 .

19) W. C. Bray u. F. L. Hunt, Journ. Aner Chem. Soc. 33, 781 (1911).

30) S. P. L. Sörenseu, Zeitschr. f. physiol Chem. 103, 164 (1918). 
wegen der Unsicherheit der Werte firr $\propto$ und $\beta$ keinen Anspruch auf grobe Genauigkeit machen. Eine genaue Messung besonders der hochprozentigen Gelatinelösungen ist zudem mit Schwierigkeiten verknüptt, weil das Durchmischen und Auffüllen im MeBkölbchen durch die Zahigkeit und die Schaumbildung, die auch bei längerem Stehen nicht verschwindet, be hindert wird. Ein höheres Erwärmen vor der Messung mub wegen der dadurch eintretenden Aenderung der Leitfähigheit vermieden werden. was schon $F$. Rettig ${ }^{21}$ ) bei Gelatine-Salzgemischen beobachtete. Man könnte zunächst erwarten, das auch die Zâhigkeit, auf die in Formel (4) keine Rücksicht genommen ist, auf die Leitfähigkeit von Gelatine-Salzsầurelossungen. von Einflub sein müBte. Wir konnten häufig beobachter, dab das nicht der Fall ist. Lösungen mit höherem Gelatinegehait sind anfangs noch flüssig, werden aber bei längerem Stehen in Thermostaten fest. Dieses Erstarren hat nicht den geringsten Einflub auf die Leitfăhigkeit, worauf jüngst auch schon $\mathrm{F}$. Rettig (loc cit.) hingewiesen hat.

So ergab sich 2. B. bei einer dreiprozentigen Gelatue- 0,05n $\mathrm{HCl}$-Losung, wie aus den folgenden Zahlen zu ersehen, nach 48 stündigem Stehen bei $25,0^{\circ}$ keine Aenderung der Leitfahigkeit, obwohl die Lösung anfangs vollkommen flüssig, später abet vollkommen er* starrt war.

\begin{tabular}{|c|c|c|c|}
\hline \multirow{2}{*}{$\begin{array}{l}\text { Rhaostaten- } \\
\text { widerstand }\end{array}$} & \multicolumn{3}{|c|}{ Steliung des Schleifkontakts am } \\
\hline & 16.11 .1920 & 17.11 .1920 & 18.11 .1920 \\
\hline $\begin{array}{l}160 \\
180 \\
200 \\
220\end{array}$ & $\begin{array}{c}\text { flussig } \\
557,0 \\
526,5 \\
499,6 \\
475,0\end{array}$ & 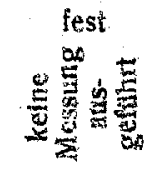 & $\begin{array}{l}\text { fest } \\
556,5 \\
526,5 \\
499,4 \\
475,1\end{array}$ \\
\hline
\end{tabular}

Um, wenn möglich, einer Aufschuß über die chemische Einheitlichleit Jer Gelatine $2 u$ gewinnen, wurde versucht, durch Membranflttration wässeriger Gelatinelösungen eine wenn auch nur teilweise Trennung der kristallolden und kolloiden Bestandteile bzw. der niedtig molekularen und hochmolekularen hetbelzuführen, oder doch wenigstens Anzeichen dafür zu gewinnen, ob in der Gelatine eine chemisch einheitliche Stibstanz oder ein Gemisch vorliegt.

Eine saubere Membranfiltration der Gelatine ist bis jetzt mit großen Schwierigkeiten verknüpft, bei allen Gelatinelösungen von etwa 0,5 Proz. an aufwärts ist nach kurzem. Filtrieren das Filter für die Flüssigkeit nut noch schwer

21) F. Rettig, Koll.-Zeitschr. 2\%, 165 (1920). passierbar. Auf dem Boden bildet sich eine Kleisterschicht; auch wein man diese durch Abpinseln inmer wieder beseitigt, gelingt es doch nicht, die Poren offen zu halten. Warme Lösungen von $30^{\circ}$ und höher lassen sich besser filtrieren, am schlechtesten filtrierbar sind die Lösungen bei Zimmertemperatur und tiefer. Heiße Lösungen von $60^{\circ}$ in nicht zu großer Konzentration laufen glatt durchs Filier, ohne einen Rückstand $2 u$ hinterlassen. Bei starkem Unterdruck verstopfen sich die Poren des Filters besonders schnell. Diese Erscheinungen zeigen sowohl weitporige 3 Sek.- Filter ${ }^{22}$ ) als engere. Um das Verstopfen der Poren und die. Bildung der kleistrigen Gelatineschicht nach Mögiichkeit zu vermeiden, wurde in eine,n Apparat mit vertikaistehenden Filtern und ganz schwacher Saugheberwithung (etwa $3 / 4 \mathrm{~m}$ Wassersaule) unter gleichzeitigem Durchrühren der Gelatinelösung mehrere Filtrationsversuche ausgeführt. Trotz der sehr geringen Saugwirlung (entsprechend geritge Filtriergeschwindigkeit) war das Filter innerhalb 15 Stunden wieder mit einer zähen Gelatineschicht belegt und fast unpassierbar gemacht.

Ausgehend von dem Gedanken, vielleicht durch Schütteln dis Bildung von Gallertelementen $z u$ verbindern, haben wir eine ganze Reihe von mehrstündigen Schüttelversuchen ausgeführt. Diarch vorhergehendes Schütteln wird das Filtrieren ganz waseritlich erleichtert, besonders insofern, als ein Verstopfen des Filters, besonders bei niederen Temperaturen (unterhalb $15^{\circ}$ ) kaum beobachtet wurde. Man kann sogar aus einer einprozentigen Gelatinelösung 96 Proz. Flissigkeit abifitrieren. Das Filtrat enthält stets mur noch geringe Menge Gelatine unter 10 Proz. Sichere Anhaltspunkte, ob auf diese Weise die Gelatine sich in mehrere Substanzen trennen läBt, haben sich bis jetxt nicht ergeben

$$
\text { Versuche mit } \beta-G \text { lutin. }
$$

Da das nicht miehr gelatinierende $\beta$-Glutin, welches man durch längeres Kochen wässeriger Gelatinelösungen erhält, im allgemeinen als Spaltungsprodukt der Gelatine angesehen wird, lag es nahe, seine physikalisch-chemischen und kolloidchemischen Eigenschaften mit den oben besprochenen der Gelatize zu vorgleichen.

22) Filter, das in dem Z sigm ondy'schen Apparat Utber dem Vakuum der Wasserstrablpumpe be $80 \mathrm{qcm}$ filtrierende Flache $100 \mathrm{~cm}$ in 3 Sekunden durchflieben laBt. R. Zsigm ondy a. G. Jander. Zeirschr f. ahai. Chem. 58, 243, (1919). R. Zsigmondy u. W. B a c hma n n, Zeitschr. f. anorg. Chem. 103, 119 (1918). 
Wir stellten unsete Prăparate in der üblichen Weise durch längeres Kochen einer ca. zehnprozentigen Gelatinelösung in einem Kolben aus Jenaer Glas am Rückflueikühler dar. Die ursprünglich klate, bei Zimmertemperatur zu einer festen Gallerte erstarrte Lösung hatte, nachdem sie ungefahr 70 Stunden gekocht war, viele ihrer physikalischen Eigenschaften völlig geändert. Sie hatte sich bräunlich gelb gefärbt und ihr Gelatinierungsvermögen völlig eingebüBt; bei Filtrieren durch ein Viersekundenfilter lief die Lösung $z$ unächst glatt durch, nachdem aber etwa die Hälfte abfiltriert war, lieb die Piltriergeschwindigkeit merklich nach, bis schlieblich nichts mehr die Membran passierte. Während aber bei Gelatinelösungen entsprechender Konzentration sich auf der Membran ein Kleister angesammelt hatte (siehe oben), trat bei $\beta$-Glutinlösungen eine Gallertabscheidung aut dem Filter nicht ein (vgl. die Filtration des durch längeres Kochen gewonnenen $\boldsymbol{\beta}$-Glutin-Präparates II).

Ganz analog wie bei der Gelatine stellten wir auch hier Gemische mit 0,05 n Salzsäure dar und bestimmten die Wasserstoffionenkonzentration durch potentiometrische Messungen.

\section{Tabelle IV.}

Wasserstoffionenkonzentration in gekochter $\mathrm{Ge}$ latine- $0,05 \mathrm{n}$ Salzsäuregemischen bei $25,0^{\circ}$, $n$ und $m$ : Mole Salzsäure und $g$ Gelatine im Liter. $\left[\mathrm{H}^{+}\right]_{\text {ber. }}$ nach Formel (5).

\begin{tabular}{c|c|c|l|l}
\hline $\mathbf{n}$ & $\mathbf{m}$ & $\boldsymbol{\pi}$ & \multicolumn{1}{c}{$\left[\mathrm{H}^{+}\right]_{\text {get. }}$} & \multicolumn{1}{c}{$\left[\mathrm{H}^{+}\right]_{\text {ber. }}$} \\
\hline $\mathbf{0 , 0 5}$ & 10 & 0,4163 & 0,0454 & 0,0396 \\
0,05 & 20 & 0,4275 & 0,0293 & 0,0293 \\
0,05 & 30 & 0,4351 & 0,0218 & 0,0190 \\
$\mathbf{0 , 0 5}$ & $\mathbf{7 0}$ & 0,5419 & 0,000340 & 0,000340
\end{tabular}

Wie man sieht, unterscheidet sich das Sãurebindungsvermögen dieses Produktes nicht wesentlich von dem der ursprünglichen Gelatine.

Die Gleichung (3) entsprechende Formel würde lauten

$$
[\mathrm{H}+]=-\frac{1}{2}\left(\frac{\mathrm{m}}{961}-\mathrm{n}+0,000158\right)
$$

$+\sqrt{\frac{1}{4}\left(\frac{m}{961}-n+0,000158\right)^{2}+n .0,000158}$.

Eine weitgehende Aenderung der Gelatine scheint also hiernach noch nicht stattgefunden zu haben.

Desgleichen wurde die spezifische Leitfähig. keit von $\beta$-Glutin- $0,05 \mathrm{n}$. Salzsäuregemischen gemessen. Das hierzu verwandte $\beta$-Glutinprăparat II war durch 150 stündiges, also
Iängeres Kochen, hergestellt, und unterschied sich von dem ersten dadurch, dab es durch 4-Sekundenfilter glatt filtriert werden konnte, ohne irgendwelchen Rückstand zu hinterlassen.

Fig. 4 und Tabelle $\mathrm{V}$ veranschaulicht die $\mathrm{Ab}$ nahme von $*$ mit zunehmendem Gelatinegehalt.

Tabelle V.

Spezifische Leitfähigkeit $x$ von $\beta$-Glutin$0,05 \mathrm{n} \mathrm{HCl}$-Gemischen in Abhängigkeit von $\beta$-Glutingehal: $\mathrm{m}$ bei $25,0^{\circ}$

\begin{tabular}{c|c}
\hline \multicolumn{2}{c}{$0,05 \mathrm{n} \mathrm{HCl}$} \\
$\mathrm{m}$ & $\times .10^{8}$ \\
\hline 0,568 & 19,46 \\
2,840 & 18,11 \\
5,68 & 16,35 \\
11,36 & 12,87 \\
28,40 & 4,970 \\
34,08 & 4,370 \\
45,44 & 4,164 \\
51,12 & 4,126
\end{tabular}

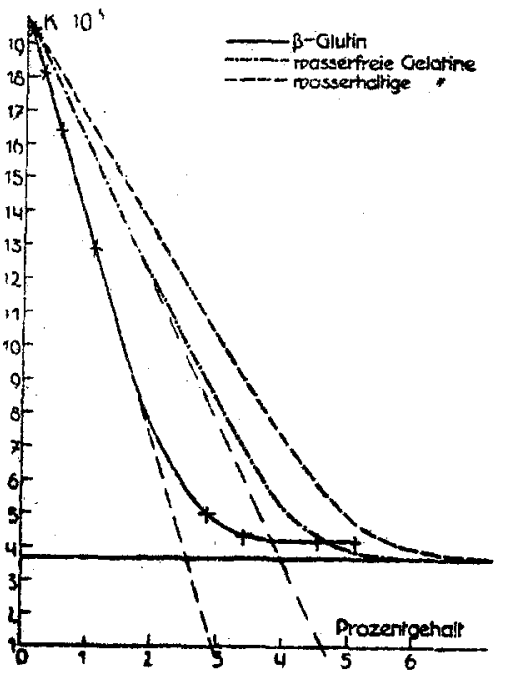

Fig. 4

Spezifische Leitfahigkeit von $\beta$ - Glutin-bzw. Gelatine$0,05 \mathrm{n} \mathrm{HCl}$ - Gemischen in Abhăngigkeit vom ProzentGehalt bei $25,0^{\circ}$.

Aus dem Vergleich der Leitfähigkeitskurve vom $\beta$-Glutin mit der von Gelatine (bezogen auf wasserfreie Gelatine) geht deutlich hervor, da $B$ das Salzsäurebindungsvermogen des $\boldsymbol{\beta}$-Glutins bei weitem größer ist als das der Gelatine. Verlängern wir den gradlinigen Teil der Kurve bis zum Schnittpunkt mit der Horizontalen durch den Punkt $1000 x=3,68$, so gibt uns dieser Schnittpunkt, wie aus folgendem hervorgeht, die $\beta$-Glutin - Konzentration an, bei we!cher alle vorhandene Salzsăure gebunden 
wăre, wenn keine Hydrolyse eintrăte. Setzt man nämlich den Grenzwert der Aequivalent-Leitfähigkeit des $\beta$-Glutinchlorids gleich dem des Gelatinechlorids, was annäherungsweise richtig sein dürfte, so ergibt sich für den Neutralpunkt, wenn keine Hydrolysse einträte, für eine $0,05 \mathrm{n}$ $\boldsymbol{\beta}$-Glutinchloridlösung, die ja im Neutralpunkt vorläge, $1000 * .20=79,21.0,9294$ nach den Daten von Seite 11 ; also $1000 *=3,68$; der Schnittpunkt liegt bei etwa 2,5 Proz.

Danach sind zur Bindung von einem $\mathrm{Mol}$ Salzsåure rund $\frac{25}{0,05}=500 \mathrm{~g} \beta$-Glutin nötig. Bei Präparat I, das, wie die Membranfiltration ergab, noch gröbere, der Gelatine ahnliche Aggregate enthält, hatte sich $M=961$ und bei Gelatine $M=885$ ergeben. Das Verbindungsgewicht des $\beta$-Glutins wäre demnach etwa halb so grob wie das der Gelatine.

DaB Präparat I und II verschiedener Natur sind, erhellt auch aus folgendem. Wie früher gezeigt, mub $\mathrm{K}$ eine lineare Funktion von $\mathrm{y}$ sein. [Siehe Formel (4)]. Berechnen wir nun die den einzelnen Leitfähigkeitsmessungen mit Präparat II entsprechenden Konzentrationen der freien Saure mit Hilfe der Konstanten K und $M$ aus Präparat $I$ und stellen ihre $A b$ hängigkeit dar, so zeigt Fig. 5, daß es sich nun nicht mehr wie bei der Gelatine um eine gradlinige Funktion handelt. $\beta$-Glutin 1 und $\beta$-Glutin II sind eben verschiedene Präparate. Dementsprechend läbt sich die Leitfähigkeit von Gemischen aus Salzsäure und $\beta$-Glutin II auch nicht annähernd aus den potentiometri- schen Messungen der entsprechenden Lösungen mit $\beta$-Glutin I berechnen.

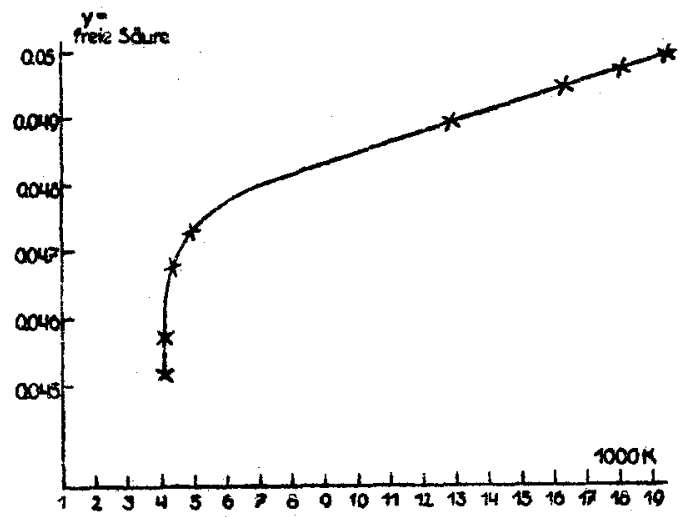

Fig, 5

Spezifische Leitfahigkeit von Praparat II und ungebundene Säure von Prăparat I bei $25,0^{\circ}$

Trübungserscheinungen, wie sie bei Gelatine-Salzsäuregemischen im isoelektrischen Punkte auftreten, konnten bei ha bprozentigen $\beta$-Glutinlösungen mit entsprechendem wechselnden Salzsäurezusatz nicht beobachtet werden. Im Ultramikroskop zeigte das Präparat selbst in verhältnismäBig konzentrierter Lösung nur einen schwachen blaBbläulichen Lichtkegel, der bei Sonnenlicht im Immersionsuitramikroskop auch beim Verdünnen mit Wasser sich nicht optisch auflösen lieB.

Göttingen, 6. Oktober 1921. Institut für anorganische Chemis

\section{Technische Bestimmung des kolloiden Anteils in Wolframpulvern.}

Vor mehreren Jahren wurde ich ersucht, ein Gutachten darüber abzugeben, ob in fünf Proben Wolframpulver, die speziell zur Herstellung von Giühfäden für elektrische Lampen bestimmt waren, ein Teil des Wolframs in kolloidem Zustande enthalten sei. Im zutreffenden Falle sollte weiter ermittelt werden, welcher Prozentsatz an kolloidem Wolfram in den einzelnen Proben vorhanden sei.

$\mathrm{Zu}$ dieser Aufgabe sind zunächst folgende rein theoretischen Bemerkungen zu machen.

Die Frage, ab in den vorgelegten Proben ein kolloider Anteil enthalten und wie grob derselbe ist, hängt innig zusammen' mit der Prage nach den Grenzen des kolloiden $\mathbf{Z}$ - standes iiberhaupt. Es ist deshaib zuerst die Frage zu beantworten, ob man eine solche Begrenzung vornehmen kann und darf. Den kolloiden Zustand eines Stoffes, also hier des Wolframs, definiert man am besten nach Wo. Ostwald als einen solchen, bei welchem die spezifische Oberfläche, d.h. das Verhältnis der Oberfläche zum Volumen des Stoffes einen sehr groben Wert angenommen hat. In diesem hochdispersen Zustande setzt sich nun das Wolfram aus seinem Dispersionsmittel, z. B. aus Wasser, trotz des ungeheuer groben Unterschiedes der spezifischen Gewichte der dispersen Phase und des Dispersionsmittels nur äuBerst träge ab. Je feiner verteilt das Wolfram ist, um so länger 